\title{
Modelling Harmonic Propagation in HVDC System Power Cables
}

\author{
Arshad*, Brian G. Stewart \\ Institute for Energy and Environment, Faculty of Engineering \\ University of Strathclyde, Glasgow, United Kingdom \\ *ORCID:0000-0001-8621-2773
}

\begin{abstract}
In this paper a three level Voltage Source Converter (VSC) based HVDC system is implemented and simulations are carried out to monitor the harmonic profile along HVDC cables. The harmonic profile of the sending and receiving end of the cables are analyzed under different conditions. The impact of changes in background harmonic sequence on the harmonic profile of the HVDC cables is analyzed. The obtained simulation results can be used to visualize the complete harmonic frequency spectrum of HVDC cables. The potential impact of harmonic distortion on cable insulation is discussed in terms of the correlation between harmonic frequency and the different mechanisms responsible for cable ageing and degradation such as partial discharges and electrical treeing. The simulation results presented in this paper will help to improve the understanding of the harmonic propagation within HVDC cables and the harmonic impact on cables insulation.
\end{abstract}

Keywords-VSC-HVDC, background harmonics, cables, converters.

\section{INTRODUCTION}

The deployment of Voltage Source Converters (VSCs) in HVDC and other Flexible Alternating Current Transmission System (FACTS) devices has increased many folds in the last decade due to the rapid development in semiconductor devices such as Insulated Gate Bipolar Transistors (IGBTs) [1]. VSCs offer many advantages in comparison to conventional thyristorbased converters such as interconnecting weak AC systems and renewable energy integration [2]. However, one of the main challenges in VSC HVDC networks is the presence of harmonic distortion. With the development of multilevel topologies and Pulse Width Modulation (PWM) techniques, the harmonic distortion has been reduced to a certain extent. However, the issue cannot be ignored completely due to the harmonic transfer characteristics of converters. During the AC/DC conversion process, harmonics are generated which may influence the operation of HVDC networks in different ways. But apart from harmonic generation of the converters, the presence of background harmonics in neighboring AC systems present significant risk [3]-[5].

During steady state operation, characteristic harmonics may be present at both $\mathrm{AC}$ and $\mathrm{DC}$ sides of the converter which depends on the converter topology, modulation technique and switching frequencies. However, during unbalance conditions, or in the presence of background distortion in the $\mathrm{AC}$ system, non-characteristic harmonics are generated at both $\mathrm{AC}$ and $\mathrm{DC}$ sides. Furthermore, if the neighboring AC systems operate at different frequencies, the characteristic harmonics of one converter will act as inter-harmonics for the neighboring converter and result in a different frequency spectrum at the $\mathrm{AC}$ side [6]-[8].

Nguyen and Han [3] studied the harmonic transfer characteristics of VSC HVDC networks using switching functions. The three switching functions considered for generating harmonic spectra are the fundamental component, carrier sideband and naturally sampled PWM. Nevertheless, the presented results only investigate the changes in background harmonic amplitude during transfer from the AC to DC side and vice-versa. Jiang et al. [7] studied the harmonic transfer characteristics of LCC and VSC converters using space vector methods to demonstrate the dependency of harmonic relative frequency and phase. However, the presented rules are only valid for three phase converters with symmetrical modulation. Liu et al. [9] studied the harmonic transfer characteristics by introducing a new harmonic model of VSCs. The relationship between harmonics and converter control variables are investigated analytically. The influence of fundamental power flow as well as various operating conditions on harmonic power flow was presented. Han et al. [10] investigated the harmonic transfer characteristics of VSC based multi-terminal HVDC networks. Detailed study of DC side harmonics revealed that inter-harmonics are produced at the DC side and are more dominant in the low frequency range. Furthermore, due to the inter-harmonic resonance, the harmonic transfer can be magnified. Additionally, the DC link capacitors of the threelevel VSCs will cause low frequency inter-harmonics in the AC systems. This phenomenon could be more visible in the case of negative sequence harmonics due to smaller frequencies.

The inter-harmonics in VSC based networks have been predicted using eigenvalue analysis and the DC equivalent impedance was devised to analyze harmonic transfer across the converters in [11]. The authors argued that the eigenvalue analysis is easy to implement in multi-terminal systems where many VSCs are used. Xu et al. [4] investigated the resonance phenomenon excited by the existing background harmonics in multi-terminal networks. The effect of AC background harmonics on DC side resonance and its effect on the remote $\mathrm{AC}$ systems was examined. It has been shown that harmonic transfer and propagation is more evident when the DC side resonates at the corresponding background harmonics frequencies. To eliminate the harmonic transfer across the VSC converters, the idea of AC/DC decoupling has been proposed in [12]. However, the proposed method requires DC voltage coupling in combination with $\mathrm{AC}$ current tracking applied to individual VSCs resulting in a complicated control system.

It is important to understand the correlation between harmonic distortion and the different mechanisms responsible 
for power cable ageing and degradation. There is limited available literature on the impact of harmonic distortion on the long-term operation and useful life of HVDC cables. It has been reported in [13], [14] that the presence of harmonic distortion in HVDC cables is responsible for the initiation of different insulation degradation mechanisms such as water treeing, electrical treeing, space charge and partial discharges. Electrical tree initiation and growth under DC superimposed AC has been investigated in [15] which shows that the tree growth in insulation depends on the AC component. The effect of harmonic frequency on partial discharge voltage magnitude and discharge time has also been reported in [16]. The obtained results showed that increasing the harmonic frequency up to 2.5 $\mathrm{kHz}$ result in doubling the discharge voltage while the reducing the discharge time by $40 \%$.

Understanding the process of harmonic transfer and propagation along the HVDC network is therefore very important for reliable operation and understanding the impact of possible insulation degradation within the system. It has been shown in [17], [18] that a second order background harmonic on the AC side transfer to the DC side as the fundamental component while a fundamental harmonic on the DC side transfers as a second harmonic and $\mathrm{DC}$ component on the $\mathrm{AC}$ side. The harmonic transfer characteristics of LCC and VSC based HVDC system have been studied in literature. However, the existing literature of VSCs converter is mostly based on analytical calculations and does not consider the various possibilities of background harmonics propagation. In this paper, a systematic and detailed analysis of background harmonics propagation in VSC HVDC cables is studied with focus on background harmonic sequence. The paper is organized as follows: section 2 presents detailed description of the simulation model followed by results and discussions in section 3 with conclusion in section 4 .

\section{TEST SYSTEM}

To simulate the propagation of background harmonics in an HVDC network, a Simulink model was setup consisting of two AC systems connected via an HVDC cable as shown in Figure 1. The test system is based on the Hydro-Quebec HVDC systems model in MATLAB/Simulink. The network consists of two $230 \mathrm{kV}, 2000 \mathrm{MVA}, 50 \mathrm{~Hz}$ AC systems connected via a $\pm 100 \mathrm{kV}, 200$ MVA HVDC cable. The cable parameters considered are given in Table 1 . The VSC at converter station 1 is modelled as the power dispatcher while the VSC at converter station 2 acts as DC voltage regulator and reactive power controller. The VSCs are based on a three level Neutral Point Clamped (NPC) converter. The Sinusoidal Pulse Width Modulation (SPWM) technique is adopted by converters where the frequency of the carrier wave is $1350 \mathrm{~Hz}$ (frequency index of 27). The converter transformers are connected in $\mathrm{Y}-\Delta$ configurations while $27^{\text {th }}$ and $54^{\text {th }} \mathrm{AC}$ shunt harmonic filters are installed at the $\mathrm{AC}$ side to minimize harmonic distortion caused by the carrier wave. Similarly, a $3^{\text {rd }}$ order harmonic filter is installed at the DC side to remove the unwanted harmonics caused by the three level NPC converter. The VI blocks in Figure 1 represent the location of current and voltage waveform measurements. The individual harmonic spectrum as well as Total Harmonic Distortion (THD) are calculated at these locations. The background harmonics can be either positive sequence (rotates with fundamental frequency), negative sequence (rotates against the fundamental frequency) or zero sequence (does not rotate).

TABLE I. HVDC CABLE PARAMETERS

\begin{tabular}{|c|c|}
\hline Length $(\mathrm{km})$ & 100 \\
\hline Resistance $(\Omega / \mathrm{km})$ & $1.39 \times 10^{-2}$ \\
\hline Inductance $(\mathrm{H} / \mathrm{km})$ & $1.59 \times 10^{-4}$ \\
\hline Capacitance $(\mathrm{F} / \mathrm{km})$ & $2.37 \times 10^{-7}$ \\
\hline No. of Pi sections & 2.0 \\
\hline
\end{tabular}

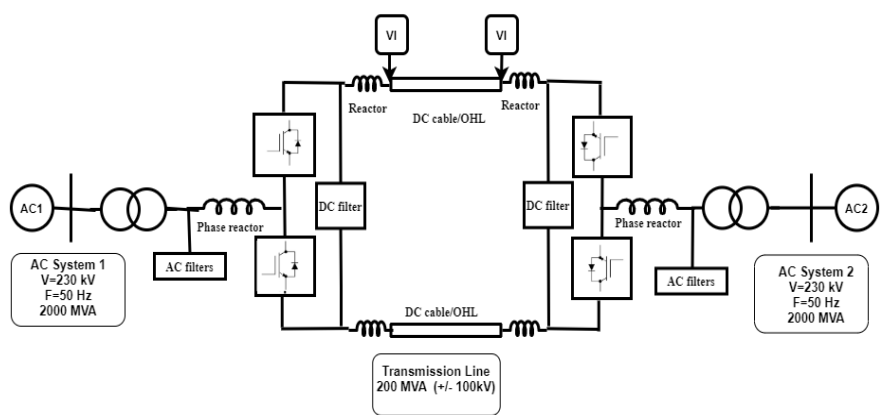

Figure 1 Schematic diagram of VSC HVDC network.

\section{SimUlation RESULTS AND DisCUSSIONS}

The system shown in Figure 1 was simulated in MATLAB/Simulink and the obtained results are presented in this section. The voltage waveform at the sending and receiving ends of the HVDC cables are presented along with their frequency spectrum under normal operation as well as in the presence of background harmonics. The frequency spectrum was obtained by taking the FFT of voltage waveforms at sending and receiving end at a sampling interval of $7.407 \times 10^{-6}$. Figure 2 and Figure 3 show the sending voltage and the corresponding frequency spectrum respectively. It is evident from Figure 3 that under normal operating conditions, zero sequence harmonics such as $3^{\text {rd }}, 6^{\text {th }}, 9^{\text {th }}, 12^{\text {th }}, 24^{\text {th }}, 27^{\text {th }}$, and $30^{\text {th }}$ are present. The dominant $27^{\text {th }}$ order harmonic is due to the carrier frequency of $1350 \mathrm{~Hz}$. Apart from characteristics harmonics, a noncharacteristics harmonic is also present at $290 \mathrm{~Hz}$ acting as sideband to the $6^{\text {th }}$ order harmonic. Similarly, the receiving end voltage and corresponding frequency spectrum are shown in Figure 4 and Figure 5 respectively. It can be seen from Figure 5 that the frequency spectrum at the receiving end is the same as the sending end in terms of harmonic order. However, there are differences in harmonic amplitudes and THD at both ends.

To further study the impact of background harmonics on the frequency spectrum of HVDC cables, background harmonics were introduced at AC system 1. Figure 6 shows the harmonic spectrum of sending and receiving ends when a positive sequence $7^{\text {th }}$ order harmonic is injected at AC system 1. The amplitude and phase angle of the background harmonic are 0.1 $\mathrm{pu}$ and $0^{\circ}$ respectively. It is evident from Figure 6 that in the presence of background harmonic the THD as well as harmonic spectrum changes. The THD measured at the sending end is $2.70 \%$ while that of receiving end is $2.47 \%$. Furthermore, apart from the characteristics zero sequence harmonics such as $3^{\text {rd }}, 6^{\text {th }}$, $9^{\text {th }}$ and so on, inter-harmonics are present as well. The interharmonics exist as sidebands around $3^{\text {rd }}$ and $6^{\text {th }}$ harmonic notably at 140, 160, 295 and $305 \mathrm{~Hz}$. Comparing the amplitudes 
of individual harmonics with and without background harmonics, Figure 3, Figure 5 and Figure 6 reveal that with the introduction of positive sequence $7^{\text {th }}$ order harmonic, the amplitude of the $6^{\text {th }}$ order harmonic increases from 0.73 to 1.47 at the sending end and from 0.54 to 0.90 at the receiving end.

Figure 7 shows the harmonic spectrum of sending and receiving ends of HVDC cables in the presence of a negative sequence $5^{\text {th }}$ order harmonic at AC system 1 . Similar to the positive sequence background harmonic, the negative sequence harmonic has a magnitude of $0.1 \mathrm{pu}$ and $0^{\circ}$ phase angle. Comparing these results with Figure 3 and Figure 5 shows that with the addition of background harmonics in AC system 1, the THD as well as the harmonic spectrum changes. The amplitude of inter-harmonics around $3^{\text {rd }}$ and $6^{\text {th }}$ order harmonics changes with change in background harmonic sequence as can be noted from Figure 6 and Figure 7.

Figure 8 shows the frequency spectrum of sending and receiving ends of the HVDC cable in the presence of a zerosequence $3^{\text {rd }}$ order harmonic having amplitude of $0.1 \mathrm{pu}$ and phase angle of $0^{\circ}$. The characteristics harmonics present in this case are almost similar to Figure 6 and Figure 7 except $18^{\text {th }}$ order harmonic in the case of positive sequence background harmonic. However, the inter-harmonics profile is different specially the sidebands around $3^{\text {rd }}$ harmonic. The THD is much lower as compared to positive and negative sequence background harmonics and is almost identical to the THD under normal operating conditions. It should be noted that in Figure 6, Figure 7 and Figure 8, the harmonic components with amplitude greater than $0.10 \%$ were considered.

Figure 9 shows the relationship between HVDC cable length and THD. These results were obtained under normal operating conditions and without the presence of any background harmonics. It was observed that with increase in cable length, the THD decreases at both ends. This is due to the increase in cable impedance resulting in low propagation of background harmonics across the cables. These results indicate that the presence of background harmonics in the AC system may not be eliminated and propagate along the HVDC chain impacting the insulation of HVDC components such as bushing, transformer windings and HVDC cables.

The existing literature indicates that the presence of harmonic distortion will severely impact the insulation due to the increased activity of partial discharges, electrical treeing and space charge. However, more work is needed to establish a correlation between harmonic distortion (power quality), partial discharges, and electrical treeing in HVDC cables. It can also be observed from the simulation results that the magnitude of certain harmonics such as $6^{\text {th }}$ order, changes as it propagates from sending end to receiving end while other does not change much for example $27^{\text {th }}$ in this case. This different propagation behaviour of harmonics makes it challenging to understand the impact on cable insulation. Therefore, extensive work is needed to understand the harmonic propagation along the cable length as well as its relationship in relation to different harmonics and TDH with respect to partial discharges and electrical treeing.

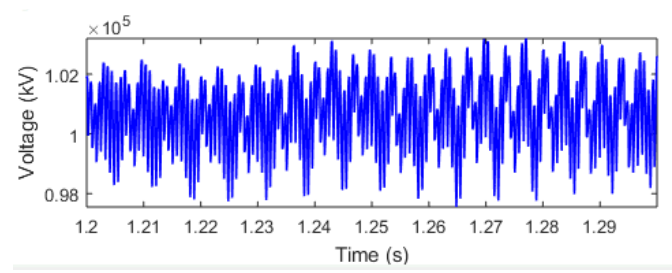

Figure 2 Voltage waveform at the sending end of HVDC cable.

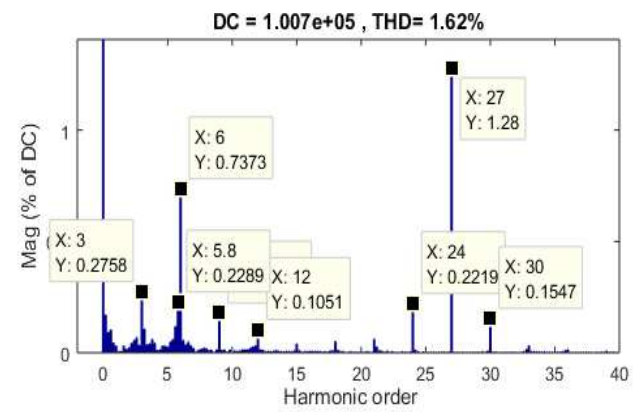

Figure 3 Harmonic spectrum of sending end voltage.

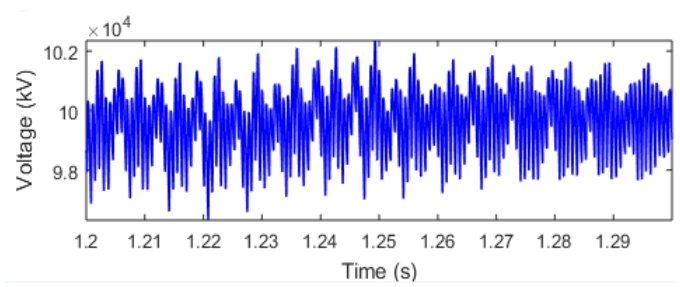

Figure 4 Voltage waveform at the receiving end of HVDC cable.

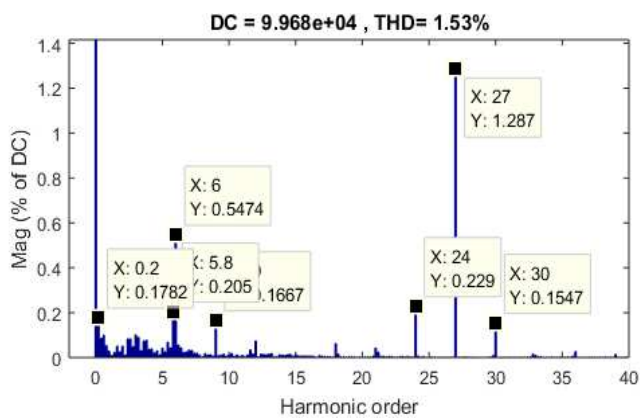

Figure 5 Harmonic spectrum at the receiving end

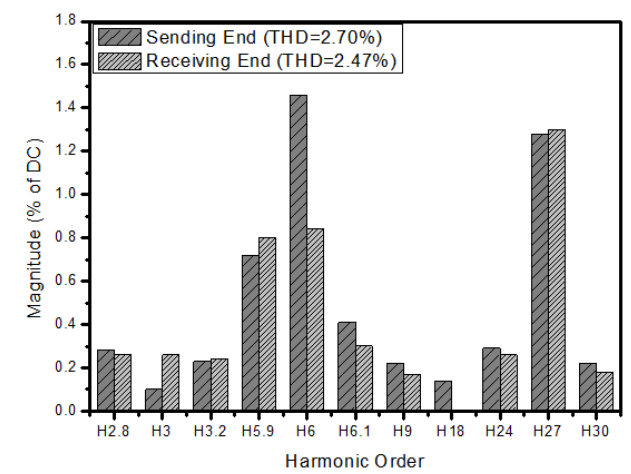

Figure 6 Harmonic spectrum at sending and receiving end in the presence of a positive sequence background harmonic. 


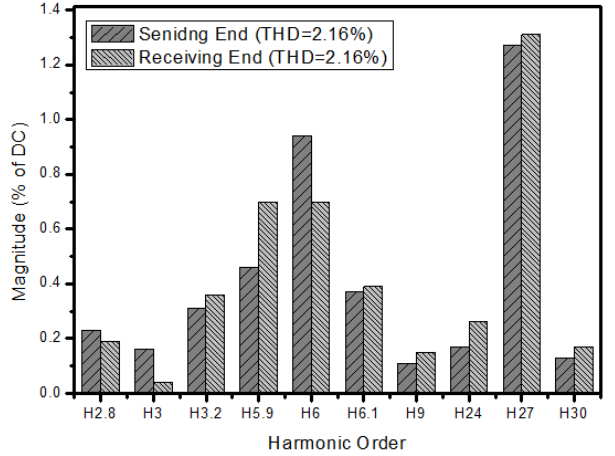

Figure 7 Harmonic spectrum at sending and receiving end in the presence of a negative sequence background harmonic.

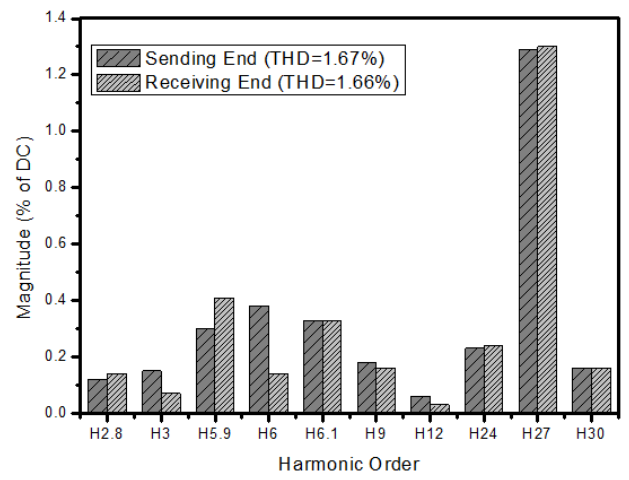

Figure 8 Comparison of harmonic spectrum at sending and receiving end in the presence of a zero-sequence background harmonic.

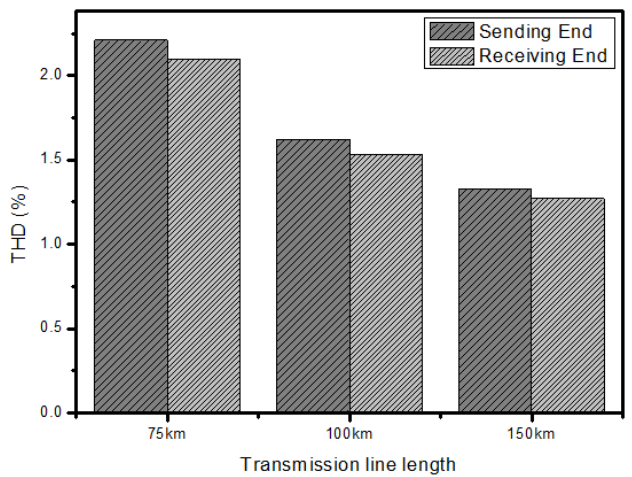

Figure 9 Relationship between cable length and THD.

\section{CONCLUSION}

This paper presents some harmonic simulation studies in a VSC HVDC network. The effect of background harmonics in neighboring AC systems on the harmonic profile of the HVDC cable is studied. It is evident from the simulation results that background harmonics are not eliminated by the filters and reactors and eventually propagate to the cables. It was also concluded that the background harmonic sequence effects the harmonic spectrum at the sending and receiving end of HVDC cables. The impact of positive sequence background harmonics was noted to be more prominent as compared to negative and zero sequence harmonics. In addition, background harmonics also result in inter-harmonics at low frequencies. In all studied cases, it was noted that $6^{\text {th }}$ order harmonic is prominent at both ends which may result in tree initiation, partial discharges and eventually cable degradation. These are preliminary results and further work is required to fully understand the consequences of background harmonic amplitude, phase and cable parameters.

\section{ACKNOWLEDGMENT}

This work was carried out at University of Strathclyde supported by EPSRC grant EP-T001445-1.

\section{REFERENCES}

[1] N. G. Hingorani, 'FACTS technology-state of the art, current challenges and the future prospects', in IEEE Power Engineering Society General Meeting, 2007, vol. 2.

[2] J. Glasdam, J. Hjerrild, L. H. Kocewiak, and C. L. Bak, 'Review on multi-level voltage source converter based HVDC technologies for grid connection of large offshore wind farms', in 2012 IEEE International Conference on Power System Technology (POWERCON), 2012, pp. 16.

[3] P. Nguyen and M. Han, 'Study on harmonic propagation of VSC-based HVDC systems', in 2014 International Conference on Power System Technology, 2014, pp. 2146-2153.

[4] D. Xu, M. Han, and A. M. Gole, 'Propagation of AC background harmonics in MMC HVdc multiterminal systems due to resonances and mitigation measures', IEEE Trans. Power Deliv., vol. 33, no. 1, pp. 229-238, 2017.

[5] W. Feng, L. Yang, F. Di, S. Guoqiang, Z. Linli, and L. Lisheng, 'Analysis of harmonic transmission characteristics in HVDC system', in 2018 2nd IEEE Conference on Energy Internet and Energy System Integration (EI2), 2018, pp. 1-6.

[6] L. Hu and R. Yacamini, 'Harmonic transfer through converters and HVDC links', IEEE Trans. Power Electron., vol. 7, no. 3, pp. 514-525, 1992.

[7] Y. Jiang and A. Ekstrom, 'General analysis of harmonic transfer through converters', IEEE Trans. Power Electron., vol. 12, no. 2, pp. 287-293, 1997.

[8] A. Testa et al., 'Interharmonics: Theory and modeling', IEEE Trans. Power Deliv., vol. 22, no. 4, pp. 2335-2348, 2007.

[9] B. Liu, Z. Du, and C. Li, 'Harmonic power flow of VSC-HVDC based AC/DC power systems', Electr. Power Syst. Res., vol. 133, pp. 355364, 2016.

[10] M. Han, P. Nguyen, and W. Yan, 'Inter-harmonics in multi-terminal VSC-based HVDC systems', J. Mod. Power Syst. Clean Energy, vol. 4, no. 2, pp. 282-291, 2016.

[11] L. Tang and B.-T. Ooi, 'Converter nonintegral harmonics from AC network resonating with DC network', in Conference Record of the 2001 IEEE Industry Applications Conference. 36th IAS Annual Meeting (Cat. No. 01CH37248), 2001, vol. 4, pp. 2186-2192.

[12] L. Tang and B.-T. Ooi, 'Elimination of "harmonic transfer through converters" in VSC-based multiterminal DC systems by AC/DC decoupling', IEEE Trans. Power Deliv., vol. 23, no. 1, pp. 402-409, 2008.

[13] G. C. Montanari et al., Diagnostics and Accelerated Life Endurance Testing of Polymeric Materials for HVDC Application. 2015.

[14] E. Corr et al., 'Partial discharge testing of defects in dielectric insulation under DC and voltage ripple conditions', CIGRE Sci. Eng., vol. 11, pp. 117-125, 2018.

[15] H. Zheng, G. Chen, and S. M. Rowland, 'The influence of AC and DC voltages on electrical treeing in low density polyethylene', Int. J. Electr. Power Energy Syst., vol. 114, p. 105386, 2020.

[16] W. Zhao, W. H. Siew, M. J. Given, E. Corr, Q. Li, and J. He, 'Assessment of HDPE aged under DC voltage combined with AC harmonic stresses of various frequencies', IEEE Trans. Dielectr. Electr. Insul., vol. 24, no. 2, pp. 1189-1196, 2017.

[17] E. V. Larsen, D. H. Baker, and J. C. McIver, 'Low-order harmonic interactions on AC/DC systems', IEEE Trans. Power Deliv., vol. 4, no. 1, pp. 493-501, 1989.

[18] J. D. Ainsworth, 'Core saturation instability in Kingsnorth HVDC link', CIGRE SC14 Winn., 1977. 